\title{
PERIODIC STRATEGIES: A NEW SOLUTION CONCEPT AND AN ALGORITHM FOR NONTRIVIAL STRATEGIC FORM GAMES
}

\author{
V. K. OIKONOMOU,*; and J. JOST ${ }^{*, \dagger}, \S$ \\ *Max Planck Institute for Mathematics in the Sciences, \\ Inselstrasse 22, 04103 Leipzig, Germany \\ ‘Santa Fe Institute, New Mexico, USA \\ tvoiko@physics.auth.gr; v.k.oikonomou1979@gmail.com \\ §jost@mis.mpg.de
}

Received 30 January 2017

Revised 15 August 2017

Accepted 17 August 2017

Published 22 September 2017

\begin{abstract}
We introduce a new solution concept, called periodicity, for selecting optimal strategies in strategic form games. This periodicity solution concept yields new insight into nontrivial games. In mixed strategy strategic form games, periodic solutions yield values for the utility function of each player that are equal to the Nash equilibrium ones. In contrast to the Nash strategies, here the payoffs of each player are robust against what the opponent plays. Sometimes, periodicity strategies yield higher utilities, and sometimes the Nash strategies do, but often the utilities of these two strategies coincide. We formally define and study periodic strategies in two player perfect information strategic form games with pure strategies and we prove that every nontrivial finite game has at least one periodic strategy, with nontrivial meaning nondegenerate payoffs. In some classes of games where mixed strategies are used, we identify quantitative features. Particularly interesting are the implications for collective action games, since there the collective action strategy can be incorporated in a purely noncooperative context. Moreover, we address the periodicity issue when the players have a continuum set of strategies available.
\end{abstract}

Keywords: Game theory; solution concepts; strategic form games; mathematical economics.

\section{Motivation for Periodicity and Periodic Strategies-A Noncooperative Concept}

We introduce a new concept in game theory that is an inherent characteristic of every nontrivial, finite action-player, simultaneous, strategic form game with or without perfect information. Here, nontrivial means nondegenerate payoffs for the

This is an Open Access article published by World Scientific Publishing Company. It is distributed under the terms of the Creative Commons Attribution 4.0 (CC-BY) License. Further distribution of this work is permitted, provided the original work is properly cited. 
players. Should a game have degenerate pay-offs, it can be perturbed to one with nondegenerate payoffs.

We shall call this new mathematical concept "periodicity" and we shall describe both the mathematical implications and its applications in specific games. For this periodicity concept, noncooperativity plays an essential role. The key aspect is that each player tries to maximize his own payoff, by observing and predicting which action of his opponent will make his payoff maximized. The situation is noncooperative as each player tries to maximize his own payoff, but there is an important difference to standard game theory. For finding periodic strategies, each player "scans" his opponent's actions, builds hierarchical belief systems on these actions, by assigning corresponding probabilities and investigates which of his opponent's strategies will maximize his own payoffs. While this is different from standard game theory, we shall see that it is self-consistent. Moreover, it will result in payoffs that are at least as high as those reached by Nash type strategies.

Here is an example, which we will analyze in detail in a later section. Consider the game named "Test Game" appearing in Table 1, which is a two player strategic form game, played simultaneously. Player A then can use mixed strategies of the form

$$
x_{\sigma}=p a_{1}+(1-p) a_{2},
$$

and correspondingly for B:

$$
y_{\sigma}=q b_{1}+(1-q) b_{2}
$$

The utilities of the players are

$$
\begin{aligned}
& \mathcal{U}_{A}(p, q)=2 p q+50 p(1-q)+3(1-p) q+2(1-p)(1-q), \\
& \mathcal{U}_{B}(p, q)=5 p q+6 p(1-q)+10(1-p) q+5(1-p)(1-q) .
\end{aligned}
$$

This game has two pure and one mixed Nash equilibrium; the latter is given by $\left(p_{N}^{*}=\frac{5}{6}, q_{N}^{*}=\frac{48}{49}\right)$. When B plays the Nash value $q_{N}^{*}$, A's utility

$$
\mathcal{U}_{A}\left(p, q_{N}^{*}=48 / 49\right)=\frac{146}{49}
$$

is independent of his own strategy $p$, and likewise, when A plays $p_{N}^{*}$, B's utility

$$
\mathcal{U}_{B}\left(p_{N}^{*}=5 / 6, q\right)=\frac{35}{6},
$$

is independent of $q$. We now observe that there are values $\left(p_{p}^{*}=1 / 49, q_{p}^{*}=1 / 6\right)$ (with the subscript $p$ standing for "periodicity"), with the property that when

Table 1. Test game.

\begin{tabular}{lll}
\hline & $b_{1}$ & $b_{2}$ \\
\hline$a_{1}$ & 2,5 & 50,6 \\
$a_{2}$ & 3,10 & 2,5 \\
\hline
\end{tabular}


$A$ plays $p_{p}^{*}$, then his utility

$$
\mathcal{U}_{A}\left(p_{p}^{*}=1 / 49, q\right)=\frac{146}{49}
$$

now is independent of the opponent's value $q$. Similarly, B's $\mathcal{U}_{B}\left(p, q_{p}^{*}=1 / 6\right)=\frac{35}{6}$ is independent of what A does. We note that the utilities (4) and (6) agree, and the same holds for the utilities of B.

Two insights emerge from this example. First, players can seek equilibria or stationary values of their utility consistently with respect to either variable, the own action or that of the opponent. In one case, we obtain the Nash equilibrium, where it does not matter what one plays as long as the opponent sticks to his action. In the other case, we obtain an equilibrium where it does not matter what the opponent does as long as a player sticks to his own action. The latter seems better than the former. In terms of market economics, this could be of some importance, since each player who adopts some mixed strategy, by choosing to play such a strategy can earn the optimal payoff (equal to Nash), without depending on the opponents actions. As we shall analyze in more detail, this depends on optimizing not with respect to the own action, but with respect to that of the opponent. This may seem strange, like wishful thinking, but as we shall see, when both players consistently do that, they will do at least as well as when playing Nash. In fact, typically, as in this example, the equilibrium utilities are the same.

More generally, we shall see that through such a process, cycles emerge, as in the rationalizable strategies of Bernheim [7] and Pearce [8]. When trying to compute the Nash value, a player optimizes his own strategy for any action of the opponent. When that the process is iterated, the players will arrive at a cycle of rationalizable strategies, and that latter class includes the Nash equilibria. Conversely, as we shall see, for arriving at the values $p_{p}^{*}, q_{p}^{*}$, each player asks for that strategy of the opponent that is best for his current action. That is, A optimizes not his own action, but that of his opponent B. When B then takes that strategy as his starting point and computes that response of $\mathrm{A}$ that is best for him, that is, B when playing that strategy, we arrive at a new strategy of A, and the process can be repeated and iterated.

The fundamental concept in noncooperative game theory [1-6], the Nash equilibrium, is one of the most widely and commonly used solution concepts that predict the outcome of a strategic interaction in the social sciences. A pure-strategy Nash equilibrium is an action profile with the important property that no single player can obtain a higher payoff by deviating unilaterally from this strategy profile. Based on the rationality of the players, a Nash strategy is a steady state of strategic interaction. In a strategic form two-player game with only finitely many actions, assumed to have nondegenerate payoffs for simplicity, there may exist both pure and mixed Nash equilibria. In a pure equilibrium, each player chooses some definite action which is the best response to the opponent's action, and conversely. Thus, no player can unilaterally change his action without decreasing his payoff. In a mixed equilibrium, instead, each player has a probability distribution for his actions which again is 
optimal in view of the opponent's distribution. A pure equilibrium can be considered as a limit case of a mixed one where all probabilities are either 0 or 1 . Mixed cases with probabilities strictly between 0 and 1 can be computed with differential calculus, by maximizing with respect to those probabilities, because the payoffs depend differentiably on them (this is a standard assumption that we shall also make implicitly).

However, as Bernheim notes in his paper [7], the Nash equilibrium is neither a necessary consequence of rationality nor a reasonable empirical proposition. Despite the valuable contributions that the Nash equilibrium offers to noncooperative games, there is a refinement, the rationalizability solution concept [10-25]. The idea of this concept is the following. An action of a player, say A, is rationalizable if it is the best response to some action of the opponent B. In turn, B's action should be the best response to some action of $\mathrm{A}$, and so on. When we iterate this in a game with only finitely many actions, then eventually, such rationalizable actions will repeat themselves, and we obtain a periodic cycle. Our periodicity concept looks similar, with the important difference, that a player no longer computes his own best response to an opponent's action, but rather seeks that opponent's action that is best for him, given his current action. Again, the process can be iterated, and we shall then arrive at periodic cycles. In strategic form games, rationalizability is based on the fact that each player views his opponent's choices as uncertain events, each player complies to Savage's axioms of rationality and this fact is common knowledge [7]. The rationalizability concept appeared independently in Bernheim's [7] and Pearce's work [8] (a predecessor of the two papers was Myerson's work [9]). Subsequently, the rationalizability solution concept has been analyzed and refined in various games, both static and dynamic. For an important stream of papers see $[10-25]$ and references therein.

The Nash equilibrium and its refinements are statements about the existence of a fixed point in every game. In this paper, we shall present another mathematical property of finite player, finite actions, simultaneous strategic form games, which we shall call periodicity. Periodicity is a solution concept with interesting quantitative implications. The purpose of this paper is to study periodic strategies and investigate the consequences of periodicity in various cases of perfect information strategic form games. The terms "periodic" and "periodicity" indicate that there exist self-maps $\mathcal{Q}$ of the players' strategy spaces with $\mathcal{Q}^{n}=1$ for some $n \in N$. The rationalizable strategies of Bernheim and Pearce are also periodic in that sense. In our case, periodic strategies arise from an optimization scheme that is different from that underlying the rationalizable ones.

\section{Periodic Strategies in Strategic Form Games-Definitions-Pure Strategies Case}

\subsection{Introduction to the periodicity concept}

We restrict our present study to simultaneous, strategic form games with two players $\mathrm{A}$ and $\mathrm{B}$, in the context of perfect information, assuming that the game is played only 
once and also that each player has only finitely many actions available. We start with pure strategies only, before including mixed actions in a later section. The strategic form game is then defined by:

- The strategy spaces of players $A, B$, denoted by $\mathcal{M}(A)=\left\{a_{1}, a_{2}, a_{3}, \ldots, a_{N}\right\}$ and $\mathcal{M}(B)=\left\{b_{1}, b_{2}, b_{3}, \ldots, b_{N}\right\}$ (for simplicity, we shall usually assume that $N=2$, i.e., that each player has only two choices for his actions), and

- the payoff functions $\mathcal{U}_{i}: \mathcal{M}(A) \times \mathcal{M}(B) \rightarrow \mathbb{R}, i=A, B$. We shall assume, for simplicity again, that they are nondegenerate in the sense that different actions yield different pay-offs.

We then have the periodicity algorithm

- Start from Player A and his first action $a(0)$. Seek that action $b(1)$ available actions $b_{1}, b_{2}, b_{3}, \ldots, b_{N}$ of $\mathrm{B}$ for which the corresponding payoff of player $\mathrm{A}$ $\mathcal{U}_{A}(a(0), b(1))$ is maximized, that is, larger than the payoff $\mathcal{U}_{A}(a(0), b)$ for any other action $b \neq b(1)$. (Recall that we assume nondegeneracy of payoffs, so there is a unique such $b(1)$.)

- For the action $b(1)$ found in the previous step, now seek that action $a(1)$ that maximizes B's payoff, that is, $\mathcal{U}_{B}(a(1), b(1))>\mathcal{U}_{B}(a, b(1))$ for any other $a \neq a(1)$.

- When iteratively actions $a(k), b(k)$ have been chosen, seek that $b(k+1)$ for which $\mathcal{U}_{A}(a(k), b(k+1))>\mathcal{U}_{A}(a(k), b)$ for any $b \neq b(k+1)$, and then that $a(k+1)$ for which $\mathcal{U}_{B}(a(k+1), b(k+1))>\mathcal{U}_{B}(a, b(k+1))$ for any other $a \neq a(k+1)$.

- Continue until $a(k)=a(\ell)$ or $b(k)=b(\ell)$ for some $\ell<k$.

Since at each step, by nondegeneracy, the selected action of the opponent is unique, the procedure will then repeat itself, that is, become periodic. Hence the name "periodic solution".

\subsection{Two player, perfect information strategic form games}

Formalizing the preceding, we define two continuous maps between the strategy spaces,

$$
\varphi_{1}: \mathcal{M}(A) \rightarrow \mathcal{M}(B) \quad \varphi_{2}: \mathcal{M}(B) \rightarrow \mathcal{M}(A)
$$

They are defined in terms of the payoff functions by the following inequalities

$$
\begin{array}{ll}
\mathcal{U}_{A}\left(x, \varphi_{1}(x)\right)>\mathcal{U}_{A}\left(x, y_{1}\right), & \forall y_{1} \in \mathcal{M}(B) \backslash\left\{\varphi_{1}(x)\right\} \\
\mathcal{U}_{B}\left(\varphi_{2}(y), y\right)>\mathcal{U}_{B}\left(x_{1}, y\right), & \forall x_{1} \in \mathcal{M}(A) \backslash\left\{\varphi_{2}(y)\right\}
\end{array}
$$


We can achieve the strict inequalities here because we assume that the pay-off tables are nondegenerate. Iteratively, we obtain for any positive integer $k$

$$
\begin{aligned}
& \mathcal{U}_{B}\left(\left(\varphi_{2} \circ \varphi_{1}\right)^{k}(x), \varphi_{1} \circ\left(\varphi_{2} \circ \varphi_{1}\right)^{k-1}(x)\right)>\mathcal{U}_{B}\left(x_{1}, \varphi_{1} \circ\left(\varphi_{2} \circ \varphi_{1}\right)^{k-1}(x)\right), \\
& \forall x_{1} \in \mathcal{M}(A) \backslash\left\{\left(\varphi_{2} \circ \varphi_{1}\right)^{k}(x)\right\}, \\
& \mathcal{U}_{A}\left(\left(\varphi_{2} \circ \varphi_{1}\right)^{k}(x), \varphi_{1} \circ\left(\varphi_{2} \circ \varphi_{1}\right)^{k}(x)\right)>\mathcal{U}_{A}\left(\left(\varphi_{2} \circ \varphi_{1}\right)^{k} x, y_{1}\right), \\
& \forall y_{1} \in \mathcal{M}(B) \backslash\left\{\varphi_{1} \circ\left(\varphi_{2} \circ \varphi_{1}\right)^{k}(x)\right\} .
\end{aligned}
$$

Thus, in each step, a player seeks the opponent's action that maximizes his own payoff given his current action. When there are only finitely many strategies available, as we are currently assuming, then necessarily the strategies that occur in this chain will repeat themselves after finitely many steps. That is, after finitely many steps, the players turn into a periodic cycle that can be represented by the diagram

$$
x \stackrel{P}{\rightarrow} \varphi_{1}(x) \stackrel{P}{\rightarrow} \varphi_{2} \circ \varphi_{1}(x) \stackrel{P}{\rightarrow} \cdots \stackrel{P}{\rightarrow} x .
$$

We shall call such an action $x$ that repeats itself after finitely many steps periodic. The minimal number $n(x)$ of such steps is called the periodicity number of $x$. We denote the set of periodic actions of player $A$ by $\mathcal{P}(A)$ and those of player B by $\mathcal{P}(B)$. For periodic actions, the operator

$$
\mathcal{Q}=\varphi_{2} \circ \varphi_{1}
$$

satisfies $\mathcal{Q}^{n} x=x$. In terms of the operator $\mathcal{Q}$, the inequalities (9) become

$$
\mathcal{U}_{A}\left(\mathcal{Q}^{k}(x), \varphi_{1} \circ \mathcal{Q}^{k}(x)\right)>\mathcal{U}_{A}\left(\mathcal{Q}^{k}(x), y_{1}\right) \forall y_{1} \in \mathcal{M}(B) \backslash\left\{\varphi_{1} \circ\left(\varphi_{2} \circ \varphi_{1}\right)^{k}(x)\right\} .
$$

Likewise, for player B, we have the operator

$$
\mathcal{Q}^{\prime}=\varphi_{1} \circ \varphi_{2},
$$

In this case, the inequalities (9) take the form:

$$
\begin{aligned}
& \mathcal{U}_{A}\left(\left(\varphi_{1} \circ \varphi_{2}\right)^{k}(y), \varphi_{2} \circ\left(\varphi_{1} \circ \varphi_{2}\right)^{k-1}(y)\right)>\mathcal{U}_{A}\left(y_{1}, \varphi_{2} \circ\left(\varphi_{1} \circ \varphi_{2}\right)^{k-1}(y)\right), \\
& \left.\forall y_{1} \in \mathcal{M}(B) \backslash\left\{\varphi_{1} \circ \varphi_{2}\right)^{k}(y)\right\}, \\
& \mathcal{U}_{B}\left(\left(\varphi_{1} \circ \varphi_{2}\right)^{k}(y), \varphi_{2} \circ\left(\varphi_{1} \circ \varphi_{2}\right)^{k}(y)\right)>\mathcal{U}_{B}\left(\left(\varphi_{1} \circ \varphi_{2}\right)^{k}(y), x_{1}\right), \\
& \forall x_{1} \in \mathcal{M}(A) \backslash\left\{\varphi_{2} \circ\left(\varphi_{1} \circ \varphi_{2}\right)^{k}(y)\right\} .
\end{aligned}
$$

The last inequality can be written in terms of the operator $\mathcal{Q}^{\prime}$, as

$$
\mathcal{U}_{B}\left(\mathcal{Q}^{\prime k}(y), \varphi_{2} \circ \mathcal{Q}^{\prime k}(y)\right)>\mathcal{U}_{B}\left(\mathcal{Q}^{\prime k}(y), x_{1}\right) \quad \forall x_{1} \in \mathcal{M}(A) \backslash\left\{\varphi_{2} \circ \mathcal{Q}^{\prime k}(y)\right\}
$$

While the periodic strategies in our above sense and the rationalizable strategies are derived from different optimization schemes, those rationalizable strategies that are also periodic are particularly interesting. We will analyze some characteristic 
examples at the end of this section. Note that the procedure described by relations (8) and (14) does not stipulate that the maps $\varphi_{1}$ and $\varphi_{2}$ are best responses to some action. Take for example the first of the inequalities (8). It means that, by assuming that player A plays $x$, we seek in the action set of player B for an action $\varphi_{1}(x)$, for which the utility of player $\mathrm{A}, \mathcal{U}_{A}(.,$.$) is maximized. This is exactly the$ converse of the procedure followed when best responses are studied. Indeed, in the best response algorithm we don't presuppose that player A will play some action, but we ask, given that player B will play an action, say $b_{k}$, which action of player A maximizes his utility function $\mathcal{U}_{A}(.,$.$) .$

Let us briefly recapitulate what we just described. In the best response algorithm, we are searching player's A set of actions but in the periodic actions algorithm described by the inequalities (8), we search the set of players B action, given that A plays a specific action.

We also note that the periodicity number $n$ depends on the payoffs rather than on the number of available actions.

Theorem 1. Every finite action simultaneous two-player strategic form game contains at least one periodic action.

Proof. Since there are only finitely many actions, starting with any action $x_{*}$ of A and iteratively applying the operator $\mathcal{Q}$ will eventually lead to some $x$ that has already occurred before in the chain, that is, $\mathcal{Q}^{n}(x)=x$. But then, because $\mathcal{Q}$ is defined by the inequalities $(8), \mathcal{Q}^{n+k}(x)=\mathcal{Q}^{k} x$ for every positive integer $k$, and $x$ is periodic.

This reasoning reveals another property of the set of periodic actions in finite action games. We modify the definition of set stability from Bernheim [7] as follows:

Definition 1 (Set Stability). Consider an automorphism $\mathcal{Q}: \mathcal{M}(A) \rightarrow \mathcal{M}(A)$. In addition, let $A \subseteq A \cup B \subseteq \mathcal{M}(A)$, with $A \cap B=\varnothing$. The set $A$ is set stable under the action of the map $\mathcal{Q}$ if, for any initial $x_{0} \in A \cup B$ and any sequence $x_{k}$ formed by taking $x_{k+1} \in \mathcal{Q}\left(x_{k}\right)$, there exists $x_{K} \in A \cup B$ such that $d\left(x_{K}, x^{1}\right)<\epsilon$, with $x^{1} \in A$. For finite sets, this implies that any sequence formed by the act of the operator $\mathcal{Q}$ on elements produces an element $x_{k}$ for any initial $x_{0}$, with $x_{k}$ belonging to the set stable set $A$. An analogous definition applies for the set of actions of player B and the operator $\mathcal{Q}^{\prime}: \mathcal{M}(B) \rightarrow \mathcal{M}(B)$.

Theorem 2. Let $\mathcal{P}(A)$ and $\mathcal{P}(B)$ denote the set of periodic strategies for players $A$ and $B$. The sets $\mathcal{P}(A)$ and $\mathcal{P}(B)$ are set stable, under the action of the maps $\mathcal{Q}$ and $\mathcal{Q}^{\prime}$, respectively.

The theorem says that for any nonperiodic action $x_{0}$, we eventually arrive in the periodicity cycle of some action $x_{K}$, that is

$$
x_{0} \stackrel{P}{\rightarrow} \mathcal{Q}\left(x_{0}\right) \stackrel{P}{\rightarrow} \mathcal{Q}^{2}\left(x_{0}\right) \stackrel{P}{\rightarrow} \cdots x_{K} \stackrel{P}{\rightarrow} \mathcal{Q} x_{K} \stackrel{P}{\rightarrow} \cdots \stackrel{P}{\rightarrow} \mathcal{Q}^{n-1} x_{K} \stackrel{P}{\rightarrow} x_{K} .
$$


Proof. The proof of this theorem is contained in the proof of Theorem 1, so we omit it.

Finally, let us present two versions of the algorithm for finding periodic strategies. Here, we do not assume that the payoff table is nondegenerate. The first compact form of the algorithm is the following:

Algorithm 1. Consider a finite action simultaneous two-player strategic form game. We find periodic solutions according to the following algorithm.

Start with any actions $\xi_{0} \in \mathcal{M}(A), \eta_{0} \in \mathcal{M}(B)$ of $\mathrm{A}$ and $\mathrm{B}$.

Given $\xi_{k}, \eta_{k}$ for $k \geq 0$, determine $\eta_{k+1} \in \mathcal{M}(B)$ such that

$$
\mathcal{U}_{A}\left(\xi_{k}, \eta_{k+1}\right) \geq \mathcal{U}_{A}\left(\xi_{k}, y\right) \quad \forall y \in \mathcal{M}(B) .
$$

Given $\xi_{k}, \eta_{k+1}$, determine $\xi_{k+1} \in \mathcal{M}(A)$ such that

$$
\mathcal{U}_{B}\left(\xi_{k+1}, \eta_{k+1}\right) \geq \mathcal{U}_{B}\left(x, \eta_{k+1}\right) \quad \forall x \in \mathcal{M}(A) .
$$

Repeat with $k+1$ in place of $k$.

Stop with step $n$ when $\xi_{n}=\xi_{\ell}$ or $\eta_{n}=\eta_{\ell}$ for some $\ell<n$.

Here, is a more detailed description of the algorithm:

- Start from Player $\mathrm{A}$ and his first action $a_{1}$. Seek in the strategy space of $\mathrm{B}$, that is in the discrete set of all the available actions of $\mathrm{B}$, namely $b_{1}, b_{2}, b_{3}, \ldots, b_{N}$, and find the action $b_{j}$ for which, the corresponding payoff of player $\mathrm{A} \mathcal{U}_{A}\left(a_{1}, b_{j}\right)$ is maximized, that is, the payoff $\mathcal{U}_{A}\left(a_{1}, b_{j}\right)$ is the largest among all payoffs $\mathcal{U}_{A}\left(a_{1}, b_{i}\right)$, with $i=1,2,3, \ldots, j-1, \ldots, j+1, \ldots, N$. So with the procedure, we just described we have the chain of actions $a_{1} \rightarrow b_{j}$ for the moment. Note that this looks like a map from the strategy space of player A to the strategy space of player $\mathrm{B}$, and in fact, this describes the formal iteration step underlying periodicity.

- For the action $b_{j}$ found in the previous step, now seek that action of player A, among the available actions $a_{1}, a_{2}, a_{3}, \ldots, a_{N}$, for which the payoff $\mathcal{U}_{B}\left(a_{i}, b_{j}\right)$ of player B for the action $b_{j}$ is maximized. Suppose this occurs for the action $a_{k}$. So the chain of actions now becomes $a_{1} \rightarrow b_{j} \rightarrow a_{k}$.

- For the action $a_{k}$ found in the previous step, now seek that action of player B, among the available actions $b_{1}, b_{2}, b_{3}, \ldots, b_{N}$, for which the payoff $\mathcal{U}_{A}\left(a_{k}, b_{i}\right)$ of player A for the action $a_{k}$ is maximized. Suppose this occurs for the action $b_{m}$. So the chain of actions now becomes $a_{1} \rightarrow b_{j} \rightarrow a_{k} \rightarrow b_{m}$.

- ....

- ....

- The above procedure is repeated, alternating between A and B. Once one of the previous selected actions reoccurs, say $a_{0}$, then this action $a_{0}$ is characterized as a periodic action, and the final chain of actions is $a_{0} \rightarrow b_{j} \rightarrow a_{k} \rightarrow b_{m} \rightarrow \cdots \rightarrow a_{1}$ (with $b_{j}$ now being the response to $a_{0}$, etc.). The corresponding actions of player $\mathrm{B}$ then are also periodic. 
- Once such a periodic action and the correponding chain have been found, the algorithm comes to an end.

- The same procedure can be adopted for all the actions of player A and B.

\subsection{Periodic Nash and rationalizable strategies}

We are interested in those Nash strategies that are at the same time periodic actions. Suppose that the strategy set $\left(x^{*}, y^{*}\right)$ constitutes one of the Nash equilibria of a two player finite action simultaneous move game. Then the actions $\left(x^{*}, y^{*}\right)$ are mutually best responses for the two players. In order for a Nash strategy to be a periodic strategy, the following conditions must hold true.

Theorem 3. In a two-player finite action, simultaneous, strategic form game, a Nash strategy $\left(x^{*}, y^{*}\right)$ of a game is periodic if

$$
\begin{aligned}
& \varphi_{1}\left(x^{*}\right)=y^{*}, \\
& \varphi_{2}\left(y^{*}\right)=x^{*}
\end{aligned}
$$

with $\varphi_{1}, \varphi_{2}$ defined in such a way that the inequalities (8), (14) hold true. In addition, the periodicity number for each action is equal to one, that is $n=1$ and,

$$
\begin{aligned}
& \mathcal{Q}\left(x^{*}\right)=x^{*}, \\
& \mathcal{Q}^{\prime}\left(y^{*}\right)=y^{*} .
\end{aligned}
$$

Proof. The proof of Theorem 3 is simple, but we must bear in mind that the maps $\varphi_{1,2}$ do not yield in general the best response sets of the players involved in a game. Suppose that for the Nash strategy $\left(x^{*}, y^{*}\right)$, the relations (19) hold true. Acting on the first with the map $\varphi_{2}$, and with $\varphi_{1}$ on the second relation, we obtain the following relations:

$$
\begin{aligned}
& \varphi_{2} \circ \varphi_{1}\left(x^{*}\right)=\varphi_{2}\left(y^{*}\right), \\
& \varphi_{1} \circ \varphi_{2}\left(y^{*}\right)=\varphi_{1}\left(x^{*}\right) .
\end{aligned}
$$

Using relations (19), the equations (21) become:

$$
\begin{aligned}
& \phi_{2} \circ \phi_{1}\left(x^{*}\right)=x^{*}, \\
& \phi_{1} \circ \phi_{2}\left(y^{*}\right)=y^{*} .
\end{aligned}
$$

Hence, the Nash actions $\left(x^{*}, y^{*}\right)$ are periodic. The relations (22) can be cast in terms of the operators $\mathcal{Q}$ and $\mathcal{Q}^{\prime}$ as

$$
\begin{aligned}
& \mathcal{Q}\left(x^{*}\right)=x^{*}, \\
& \mathcal{Q}^{\prime}\left(y^{*}\right)=y^{*} .
\end{aligned}
$$

It is obvious that the periodicity number for the two actions is $n=1$.

Similarly, also rationalizable strategies can be periodic. This is the case if the rationalizability chain is identical to the periodicity cycle. In particular, this is the 
case if the rationalizability chains of belief contain actions that satisfy at every step the inequalities (8) and (14).

\subsection{Examples}

2.4.1. Games with and without periodic nash equilibria-four choices two player games

We start with Game 1A in Table 2, which is an analogue of one of the games in [7]. We shall focus on the choices of player A, but similar results hold for B's actions. Using the algorithm that the inequalities of relation (8) dictate, we can construct the periodicity cycles

$$
\begin{aligned}
& a_{1} \stackrel{P}{\rightarrow} b_{3} \stackrel{P}{\rightarrow} a_{3} \stackrel{P}{\rightarrow} b_{1} \stackrel{P}{\rightarrow} a_{1}, \\
& a_{3} \stackrel{P}{\rightarrow} b_{1} \stackrel{P}{\rightarrow} a_{1} \stackrel{P}{\rightarrow} b_{3} \stackrel{P}{\rightarrow} a_{3} .
\end{aligned}
$$

The periodicity number is $n=2$ for both actions, $a_{1}$ and $a_{3}$. For the actions that constitute a Nash equilibrium, it is not possible to construct such a cycle. Nevertheless, if we apply the algorithm (8), we obtain the following cycle:

$$
a_{2} \stackrel{P}{\rightarrow} b_{1} \stackrel{P}{\rightarrow} a_{1} \stackrel{P}{\rightarrow} b_{3} \stackrel{P}{\rightarrow} a_{3} \stackrel{P}{\rightarrow} b_{1} \stackrel{P}{\rightarrow} a_{1} .
$$

It is obvious that the cycle of the nonperiodic Nash action $a_{2}$ merges into the periodic cycle of the periodic action $a_{1}$, as predicted by Theorem 2 . We next consider the rationalizability cycles. The actions $a_{1}$ and $a_{3}$ are both rationalizable. and periodic, and the rationalizability cycles coincide with the periodicity cycles. Here, a rationalizability cycle is a cycle based on rationality, that is, acting optimally under some beliefs about the opponents actions. Indeed, such a cycle looks like

$$
\begin{aligned}
& a_{1} \stackrel{R}{\rightarrow} b_{3} \stackrel{R}{\rightarrow} a_{3} \stackrel{R}{\rightarrow} b_{1} \stackrel{R}{\rightarrow} a_{1}, \\
& a_{3} \stackrel{R}{\rightarrow} b_{1} \stackrel{R}{\rightarrow} a_{1} \stackrel{R}{\rightarrow} b_{3} \stackrel{R}{\rightarrow} a_{3} .
\end{aligned}
$$

The reasoning behind this cycle is based on this system of beliefs: Player A considers action $a_{1}$ rational if he believes that player $\mathrm{B}$ will play $b_{3}$, which is rational for player $\mathrm{B}$ if he believes that player A will play $a_{3}$. Accordingly, A will consider playing $a_{3}$ rational if he believes that player $\mathrm{B}$ will play $b_{1}$, which would be rational for player B if he believes that player A will play $a_{1}$. Therefore, we obtain a cycle of rationalizability based on pure utility maximization rationality.

Table 2. Game 1A.

\begin{tabular}{lllll}
\hline & $b_{1}$ & $b_{2}$ & $b_{3}$ & $b_{4}$ \\
\hline$a_{1}$ & 0,7 & 2,5 & 7,0 & 0,1 \\
$a_{2}$ & 5,2 & 3,3 & 5,2 & 0,1 \\
$a_{3}$ & 7,0 & 2,5 & 0,7 & 0,1 \\
$a_{4}$ & 0,0 & $0,-2$ & 0,0 & $10,-1$ \\
\hline
\end{tabular}


Table 3. Game 1B.

\begin{tabular}{lllll}
\hline & $b_{1}$ & $b_{2}$ & $b_{3}$ & $b_{4}$ \\
\hline$a_{1}$ & 0,7 & 2,5 & 7,0 & 0,1 \\
$a_{2}$ & 5,2 & 7,7 & 5,2 & 0,1 \\
$a_{3}$ & 7,0 & 2,5 & 0,7 & 0,1 \\
$a_{4}$ & 0,0 & $0,-2$ & 0,0 & $10,-1$ \\
\hline
\end{tabular}

The Nash action $a_{2}$ is not contained in such a cycle due to the fact that $\mathrm{A}$ will be forced to play $a_{2}$, and $\mathrm{B}$ would never play $b_{1}$ or $b_{3}$ as a best response to $a_{2}$. So, the Nash strategy is "forced" to be rationalizable. In this game, the non-Nash rationalizable actions are periodic actions which actually are the only periodic strategies and also the rationality cycles and periodicity cycles coincide.

We now slightly modify Game 1A and consider Game 1B in Table 3. The difference is that the Nash equilibrium payoffs are changed. In this case, the periodicity cycles of the actions $a_{1}$ and $a_{3}$ remain intact, but in this case, the Nash action $a_{2}$ is also periodic, with periodicity cycle

$$
a_{2} \stackrel{R}{\rightarrow} b_{2} \stackrel{R}{\rightarrow} a_{2} .
$$

Again, the periodicity and rationalizability cycles for the Nash action $a_{2}$ coincide.

\subsection{2. $2 \times 2$ games}

Now we analyze $2 \times 2$ simultaneous strategic form games. Consider first Game 2 in Table 4. The Nash equilibrium consists of the actions $\left(a_{2}, b_{2}\right)$. Following the reasoning of relation (8), we can construct the following periodicity cycles

$$
\begin{aligned}
& a_{1} \stackrel{P}{\rightarrow} b_{1} \stackrel{P}{\rightarrow} a_{1}, \\
& a_{2} \stackrel{P}{\rightarrow} b_{2} \stackrel{P}{\rightarrow} a_{2} .
\end{aligned}
$$

Obviously, all actions have a periodicity cycle and additionally all the periodicity numbers are equal to one in this particular game. Note that the actions that enter the Nash equilibrium are also periodic. However, the action $a_{1}$ is strictly dominated by the action $a_{2}$ for all cases, so it is not rationalizable. So we can never construct a cycle based on rationality argument for this action. Indeed, player A would never consider the action $a_{1}$ to be a rational move, since it can never be a best response.

Nevertheless, we can construct a cycle based on rationality arguments for the $a_{2}$ action. Indeed, player $\mathrm{A}$ would consider $a_{2}$ to be a rational move if he believed that

Table 4. Game 2.

\begin{tabular}{lll}
\hline & $b_{1}$ & $b_{2}$ \\
\hline$a_{1}$ & 3,5 & 0,2 \\
$a_{2}$ & 4,3 & 5,4 \\
\hline
\end{tabular}


player B would play $b_{2}$, which would be rational for player B if he believes that player A plays $a_{2}$. According to this line of reasoning we can construct the following rationalizability cycle,

$$
a_{2} \stackrel{R}{\rightarrow} b_{2} \stackrel{R}{\rightarrow} a_{2} .
$$

In this particular game, the set of periodic actions for player $A$ consists of both actions $a_{1}$ and $a_{2}$, that is $\mathcal{P}(A)=\left\{a_{1}, a_{2}\right\}$, while the set of rationalizable actions that are not Nash actions is empty. The set of Nash actions consists of the action $\left\{a_{2}\right\}$. For this particular game, the Nash equilibrium happens to be periodic.

In this game, the iterated elimination of dominated strategies results in $\left(a_{2}, b_{2}\right)$ which is the Nash equilibrium. This class of games describes competition between two firms that choose quantities that they produce, knowing that the total quantity that is made available in the market actually determines the price [26]. In this game, a periodic Nash equilibrium is the only action that remains after the iterated elimination of dominated strategies.

\subsubsection{Some standard games}

Before closing this section, we study the periodicity properties of the players available actions for the prisoner's dilemma game, the battle of sexes game and finally the matching pennies game. Let us start with the prisoner's dilemma game, Game 3 in Table 5, with $a<b<c<d$. The action $a_{1}$ is rationalizable but not periodic.

The action $a_{2}$ is periodic and the strategy $\left(a_{2}, b_{2}\right)$ contains periodic actions. Actually, the periodicity cycle in this case is

$$
a_{2} \stackrel{P}{\rightarrow} b_{2} \stackrel{P}{\rightarrow} a_{1} \stackrel{P}{\rightarrow} b_{1} \stackrel{P}{\rightarrow} a_{2} .
$$

Note that the periodicity number is $n=2$ in this case. Let us continue with the Battle of Sexes, Game 4 in Table 6. There are two Nash equilibria, $\left(a_{1}, b_{1}\right)$ and $\left(a_{2}, b_{2}\right)$, and both actions $a_{1}$ and $a_{2}$ are periodic and rationalizable. There are no nonNash strategies that are rationalizable. In this game, we always have $n=1$.

Table 5. Game 3, prisoner's dilemma.

\begin{tabular}{lll}
\hline & $b_{1}$ & $b_{2}$ \\
\hline$a_{1}$ & $\mathrm{~b}, \mathrm{~b}$ & $\mathrm{~d}, \mathrm{a}$ \\
$a_{2}$ & $\mathrm{a}, \mathrm{d}$ & $\mathrm{c}, \mathrm{c}$ \\
\hline
\end{tabular}

Table 6. Game 4, battle of sexes.

\begin{tabular}{lll}
\hline & $b_{1}$ & $b_{2}$ \\
\hline$a_{1}$ & 2,1 & 0,0 \\
$a_{2}$ & 0,0 & 1,2 \\
\hline
\end{tabular}


Table 7. Game 5, the matching pennies game.

\begin{tabular}{lll}
\hline & $b_{1}$ & $b_{2}$ \\
\hline$a_{1}$ & $1,-1$ & $-1,1$ \\
$a_{2}$ & $-1,1$ & $1,-1$ \\
\hline
\end{tabular}

Finally, in the matching pennies game, Game 5 in Table 7 . we have $n=2$ and the actions $a_{1}, a_{2}$ are both periodic and rationalizable, that is, we can construct the following cycles:

$$
\begin{aligned}
& a_{1} \stackrel{P}{\rightarrow} b_{1} \stackrel{P}{\rightarrow} a_{2} \stackrel{P}{\rightarrow} b_{2} \stackrel{P}{\rightarrow} a_{1}, \\
& a_{2} \stackrel{R}{\rightarrow} b_{2} \stackrel{R}{\rightarrow} a_{1} \stackrel{R}{\rightarrow} b_{1} \stackrel{R}{\rightarrow} a_{2} .
\end{aligned}
$$

All the actions of both players are periodic and rationalizable. There is no pure strategy Nash equilibrium. This motivates to turn to mixed strategies. This will be the subject of the next section.

Before closing this section, let us briefly comment on the case of extensive form games and periodicity. Since every perfect information extensive form game has a strategic form game representation, all arguments apply to extensive form games. The difference is that the strategic form representation of an extensive form game has many degeneracies, so we may have many periodicity cycles corresponding to a specific action. Here, however, this will not be analyzed further.

\section{Periodicity, Rationalizability and Mixed Strategies in Finite Action Simultaneous Strategic Form Games}

\subsection{Essential features of periodicity in the case of mixed strategies: Introductory remarks}

We shall now develop the periodicity concept for mixed strategies for finite action simultaneous strategic form games (again for two-player games only), and derive its consequences. For some classes of well-known games, the periodic mixed strategies yield the same payoff as the Nash strategies do. Sometimes, they yield even higher payoffs. The important difference, or advantage of such periodic strategies is that, in contrast to the situation for Nash equilibria, the payoff of a player does not depend on the action of the opponent. For Nash, the underlying rationality assumption is crucial. Each player is not only rational himself, but assumes that the opponent is rational as well, in the sense that he adopts the best response to the own action. An equilibrium where both act rationally in that sense is a Nash equilibrium. For the periodicity concept, in contrast, one assumes that the opponent chooses the best action not for himself, but for oneself. As we shall see, this is as self-consistent as the Nash rationality assumption. 
Let us now present the essential idea of the algorithm for mixed strategies, again for simultaneous mixed strategy, two-player, strategic form games with perfect information. And for simplicity, we grant each player two actions only.

A general mixed strategy for the player A then is of the following form:

$$
x_{\sigma}=p a_{1}+(1-p) a_{2},
$$

and one for B looks like

$$
y_{\sigma}=q b_{1}+(1-q) b_{2} .
$$

The crucial parameters here are the probabilities $0 \leq p, q \leq 1$. We assume that the payoffs depend differentiably on them. In fact, in game theory, it is usually assumed that the payoff for a mixed strategy is the convex combination of those for pure strategies, that is,

$$
\begin{aligned}
\mathcal{U}_{i}(p, q)= & p q \mathcal{U}_{i}\left(a_{1}, b_{1}\right)+(1-p) q \mathcal{U}_{i}\left(a_{2}, b_{1}\right)+p(1-q) \mathcal{U}_{i}\left(a_{1}, b_{2}\right) \\
& +(1-p)(1-q) \mathcal{U}_{i}\left(a_{2}, b_{2}\right) \text { for } i=A, B .
\end{aligned}
$$

The difference between periodic and Nash mixed strategies then reduces to the fact that for Nash, a player, say A, optimizes with respect to his own action and therefore, $\mathcal{U}_{A}(p, q)$ has to be differentiated with respect to his own probability $p$ to find the optimum. In contrast, for the periodic strategy, A will need to differentiate $\mathcal{U}_{A}(p, q)$ with respect to the opponent's probability $q$. Since $p$ and $q$ enter linearly in (34), when we differentiate with respect to $p$ and put the resulting expression $=0$ to find the extremum, the result will only depend on $q$ and not on $p$. That is, for Nash, the result is independent of the own action and only depends on the opponent's choice of $q$. In contrast, when we differentiate with respect to $q$ and set the result $=0$, it will no longer depend on the opponent's $q$, but only on the own $p$. We can thus turn the procedure into algorithmic form by iteratively performing such an optimization for the two players in turn. We work with the set-up defined in the beginning of this section, in particular the utility functions (34).

- Start with some value of $p$ for player A and put $\frac{\partial \mathcal{U}_{A}(p, q)}{\partial q}=0$. The solution specifies a mixed strategy action $p_{p}^{*}$ (with the subscript $p$ standing for "periodic").

- Continue with some value $q$ for player B and put $\frac{\partial \mathcal{U}_{B}(p, q)}{\partial p}=0$, leading to a mixed strategy action $q_{p}^{*}$.

- Check for both players if the following conditions hold true:

$$
\begin{aligned}
& \left.\frac{\partial \mathcal{U}_{A}(p, q)}{\partial q}\right|_{p=p_{p}^{*}}=0 \\
& \mathcal{U}_{A p_{p}^{*}, q}=\max \left(\mathcal{U}_{A}(p, q)\right), \quad \forall p, q, \\
& \left.\frac{\partial \mathcal{U}_{B}(p, q)}{\partial q}\right|_{q=q_{p}^{*}}=0 \\
& \mathcal{U}_{B}\left(p, q_{p}^{*}\right)=\max \left(\mathcal{U}_{B}(p, q)\right), \quad \forall p, q
\end{aligned}
$$


- Check if $q_{p}^{*}$ is the only value of $q$ that maximizes $\mathcal{U}_{A}\left(p_{p}^{*}, q\right)$ and also check if $p_{p}^{*}$ is the only value of $p$ that maximizes $\mathcal{U}_{B}\left(p, q_{p}^{*}\right)$.

- If so, stop; otherwise return to the beginning.

When the algorithm stops, we have found $p_{p}^{*}$ and $q_{p}^{*}$ which are mutually optimal. That means that $q_{p}^{*}$ is that action of $\mathrm{B}$ that is optimal for $\mathrm{A}$ when he plays $p_{p}^{*}$, and conversely.

\subsection{Periodicity for mixed strategies}

We now look for periodicity patterns in $2 \times 2$ games in the context of mixed strategies. As in the pure strategy case, this periodicity will be materialized by two maps $\Phi_{1}, \Phi_{2}$ that constitute the automorphisms $\mathcal{Q}=\Phi_{2} \circ \Phi_{1}$ and $\mathcal{Q}^{\prime}=\Phi_{1} \circ \Phi_{2}$. Their definition will be different from the pure strategy case. Take for example player A: The operator $\mathcal{Q}$ has the property that there exists a positive integer $n$ and some action $x_{\sigma} \in \Delta(\mathcal{M}(A))$, for which $\mathcal{Q}^{n} x_{\sigma}=x_{\sigma}$. The actions of the maps $\Phi_{1}$ and $\Phi_{2}$ are defined in the mixed strategies case as follows:

$$
\begin{aligned}
& \Phi_{1}: \Delta(\mathcal{M}(A)) \rightarrow \Delta(\mathcal{M}(B)), \\
& \Phi_{2}: \Delta(\mathcal{M}(B)) \rightarrow \Delta(\mathcal{M}(A)),
\end{aligned}
$$

where $\mathcal{M}(A)$ and $\mathcal{M}(B)$ are the available strategies space of player $\mathrm{A}$ and $\mathrm{B}$, and $\Delta(\mathcal{M}(A))$ and $\Delta(\mathcal{M}(B))$ are the probability distributions over the corresponding strategy spaces. These two maps $\Phi_{1}$ and $\Phi_{2}$ are defined such that we have for all $k \geq 1$

$$
\begin{aligned}
& \mathcal{U}_{B_{p, q}}\left(\left(\Phi_{2} \circ \Phi_{1}\right)^{k}\left(x_{\sigma}\right), \Phi_{1} \circ\left(\Phi_{2} \circ \Phi_{1}\right)^{k-1}\left(x_{\sigma}\right)\right)>\mathcal{U}_{B_{p, q}}\left(x_{\sigma_{1}}, \Phi_{1} \circ\left(\Phi_{2} \circ \Phi_{1}\right)^{k-1}\left(x_{\sigma}\right)\right), \\
& \forall x_{\sigma_{1}} \in \Delta\left(\mathcal{M}(A) \backslash\left\{\left(\Phi_{2} \circ \Phi_{1}\right)^{k}\left(x_{\sigma}\right)\right\}\right), \\
& \mathcal{U}_{A_{p, q}}\left(\left(\Phi_{2} \circ \Phi_{1}\right)^{k}\left(x_{\sigma}\right), \Phi_{1} \circ\left(\Phi_{2} \circ \Phi_{1}\right)^{k}\left(x_{\sigma}\right)\right)>\mathcal{U}_{A_{p, q}}\left(\left(\Phi_{2} \circ \Phi_{1}\right)^{k} x_{\sigma}, y_{\sigma_{1}}\right), \\
& \forall y_{\sigma_{1}} \in \Delta\left(\mathcal{M}(B) \backslash\left\{\Phi_{1} \circ\left(\Phi_{2} \circ \Phi_{1}\right)^{k}\left(x_{\sigma}\right)\right\}\right),
\end{aligned}
$$

when we consider player A. The algorithm implied by the inequalities (38) dictates that starting with a mixed strategy of player A, namely $x_{\sigma}$, and upon which we act with the map $\Phi_{1}$, we search in player's B set of probability distributions $\Delta(\mathcal{M}(B))$, in order to find which mixed strategy maximizes the expected utility of player A. This then is iterated. Accordingly, just like in the pure strategy case, it is possible that the process returns to the initial mixed strategy, $x_{\sigma}$. In that case, there is a chain of mixed strategies of the following form:

$$
x_{\sigma} \stackrel{P}{\rightarrow} \Phi_{1}\left(x_{\sigma}\right) \stackrel{P}{\rightarrow} \Phi_{2} \circ \Phi_{1}\left(x_{\sigma}\right) \stackrel{P}{\rightarrow} \cdots \stackrel{P}{\rightarrow} x_{\sigma}
$$

where as in the pure strategy case, the letter $P$ denotes the procedure described in relation (38) above. A mixed strategies for which we can find such a chain, is called 
periodic, and as in the pure strategy case, such strategies satisfy

$$
\mathcal{Q}^{n} x_{\sigma}=x_{\sigma}
$$

The last inequality of (38) then becomes

$$
\begin{gathered}
\mathcal{U}_{A_{p, q}}\left(\mathcal{Q}^{n}\left(x_{\sigma}\right), \Phi_{1} \circ \mathcal{Q}^{n}\left(x_{\sigma}\right)\right)>\mathcal{U}_{A_{p, q}}\left(\mathcal{Q}^{n}\left(x_{\sigma}\right), y_{\sigma_{1}}\right) \\
\left.\forall y_{\sigma_{1}} \in \Delta\left(\mathcal{M}(B) \backslash\left\{\Phi_{1} \circ \mathcal{Q}^{n}\left(x_{\sigma}\right)\right\}\right)\right) .
\end{gathered}
$$

Dealing with mixed strategies provides the advantage that we can use differential calculus to identify the optimizers at each step. The action of the map $\Phi_{1}$ on $x_{\sigma}$ is equivalent to the maximization of $\mathcal{U}_{A_{p, q}}$ with respect to $q$. Indeed, take for example the initial inequality of relation (38). The map $\Phi_{1}$ yields a strategy $\in \Delta(\mathrm{N}(B))$ by which the expected utility of player $\mathrm{A}$ is maximized. Hence, if we differentiate $\mathcal{U}_{A_{p, q}}$ with respect to $q$, the corresponding solution $p_{p}^{*}$ is equal to $\Phi_{1}\left(x_{\sigma}\right)$,

$$
p_{p}^{*}=\Phi_{1}\left(x_{\sigma}\right) .
$$

We shall use the property implied by relation (42) in one of the next subsections to bring out interesting features in some classes of games. The same considerations apply for player B. Thereby, the corresponding inequalities (38) for a given initial mixed strategy $y_{\sigma} \in \Delta(\mathcal{M}(A))$, now become

$$
\begin{aligned}
& \mathcal{U}_{A_{p, q}}\left(\left(\Phi_{1} \circ \Phi_{2}\right)^{k}\left(y_{\sigma}\right), \Phi_{2} \circ\left(\Phi_{1} \circ \Phi_{2}\right)^{k-1}\left(y_{\sigma}\right)\right)>\mathcal{U}_{A_{p, q}}\left(y_{\sigma_{1}}, \Phi_{2} \circ\left(\Phi_{1} \circ \Phi_{2}\right)^{k-1}\left(y_{\sigma}\right)\right), \\
& \left.\forall y_{\sigma_{1}} \in \Delta(\mathcal{M}(B)) \backslash\left\{\Phi_{1} \circ \Phi_{2}\right)^{k}\left(y_{\sigma}\right)\right\}, \\
& \mathcal{U}_{B_{p, q}}\left(\left(\Phi_{1} \circ \Phi_{2}\right)^{k}\left(y_{\sigma}\right), \Phi_{2} \circ\left(\Phi_{1} \circ \Phi_{2}\right)^{k}\left(y_{\sigma}\right)\right)>\mathcal{U}_{B_{p, q}}\left(\left(\Phi_{1} \circ \Phi_{2}\right)^{k}\left(y_{\sigma}\right), x_{\sigma_{1}}\right), \\
& \forall x_{\sigma_{1}} \in \Delta(\mathcal{M}(A)) \backslash\left\{\Phi_{2} \circ\left(\Phi_{1} \circ \Phi_{2}\right)^{k}\left(y_{\sigma}\right)\right\},
\end{aligned}
$$

for every $k \geq 1$. The inequality at the periodic value $k=n$ can be written in terms of the operator $\mathcal{Q}^{\prime}$ as

$$
\mathcal{U}_{B_{p, q}}\left(\mathcal{Q}^{\prime n}(y), \Phi_{2} \circ \mathcal{Q}^{\prime n}(y)\right)>\mathcal{U}_{B_{p, q}}\left(\mathcal{Q}^{\prime n}(y), x_{\sigma_{1}}\right) \forall x_{\sigma_{1}} \in \mathcal{M}(A),
$$

where the operator $\mathcal{Q}^{\prime}$ is given by

$$
\mathcal{Q}^{\prime}=\Phi_{1} \circ \Phi_{2}
$$

Hence, a periodic action $y_{\sigma}$ of player B satisfies

$$
\mathcal{Q}^{\prime n} y_{\sigma}=y_{\sigma}
$$

We shall now discuss some standard strategic form games for which the periodicity argument applies.

\subsection{Periodic mixed strategies in some games}

To see which games have the properties discussed in the previous sections, we look at general characteristics of the payoff matrix. The maximization of A's utility function 
$\mathcal{U}_{A p, q}$ with respect to $q$ yields

$$
p_{p}^{*}=\frac{\mathcal{U}_{A p, q}\left(a_{2}, b_{2}\right)-\mathcal{U}_{A p, q}\left(a_{2}, b_{1}\right)}{\left(\mathcal{U}_{A p, q}\left(a_{1}, b_{1}\right)+\mathcal{U}_{A p, q}\left(a_{2}, b_{2}\right)-\mathcal{U}_{A p, q}\left(a_{1}, b_{2}\right)-\mathcal{U}_{A p, q}\left(a_{2}, b_{1}\right)\right)}
$$

while the maximization with respect to $p$ yields

$$
q_{N}^{*}=\frac{\mathcal{U}_{A}\left(a_{2}, b_{2}\right)-\mathcal{U}_{A}\left(a_{1}, b_{2}\right)}{\left(\mathcal{U}_{A}\left(a_{1}, b_{1}\right)+\mathcal{U}_{A}\left(a_{2}, b_{2}\right)-\mathcal{U}_{A}\left(a_{1}, b_{2}\right)-\mathcal{U}_{A}\left(a_{2}, b_{1}\right)\right)} .
$$

The relation (47) yields the potential mixed periodic strategy, while relation (48) yields the potential Nash mixed strategy.

Now, we will exploit the fact that when the opponent plays a mixed Nash equilibrium strategy, the player's expected utility is independent of his own randomization over his own strategies, and at the same time, the utility is maximized. For example, in the case of player A, this would mean that the corresponding Nash equilibrium does not depend on $p$ but on $q$. Hence, we can built games in such a way that the mixed periodic strategies are related to the mixed Nash equilibria and then explore the consequences of such a relation.

\subsubsection{First type of games}

We take a look at $2 \times 2$ games that satisfy

$$
\begin{aligned}
& p_{p}^{*}=q_{N}^{*}, \\
& q_{p}^{*}=p_{N}^{*},
\end{aligned}
$$

where $p_{p}^{*}$ and $p_{N}^{*}$ are the mixed periodic and mixed Nash equilibria for player A and $q_{p}^{*}$ and $q_{N}^{*}$ are those for player B. Hence, it is obvious how the robustness of the corresponding expected utilities is achieved. Making use of relations (48) and (47), relations (49) impose some restrictions on the payoff matrices, which are the following:

$$
\mathcal{U}_{A p, q}\left(a_{1}, b_{2}\right)=\mathcal{U}_{A p, q}\left(a_{2}, b_{1}\right), \quad \mathcal{U}_{B p, q}\left(a_{1}, b_{2}\right)=\mathcal{U}_{B p, q}\left(a_{2}, b_{1}\right)
$$

Let us illustrate this result for the Battle of Sexes in Table 8.

The mixed Nash equilibrium for this game is $\left(p_{N}^{*}=\frac{2}{3}, q_{N}^{*}=\frac{1}{3}\right)$. If we maximize A's expected utility with respect to $q$, we obtain $\frac{\partial \mathcal{U}_{A p, q}}{\partial q}=-1+3 p$, hence the mixed periodic strategy is $p_{p}^{*}=1 / 3$, and again, this is independent of the value of $q$. The corresponding maximization procedure for player $B$ yields the mixed periodic

Table 8. Mixed strategies game 1 .

\begin{tabular}{lll}
\hline & $b_{1}$ & $b_{2}$ \\
\hline$a_{1}$ & 2,1 & 0,0 \\
$a_{2}$ & 0,0 & 1,2 \\
\hline
\end{tabular}


strategy $q_{p}^{*}=2 / 3$. Let us now examine the expected utilities of the players. The expected utility for player A for the mixed "periodic" (we shall use the term periodic even though these strategies are not periodic per se, but these strategies result from using the first three steps of the mixed strategies algorithm we described in the previous sections) strategy $p_{p}^{*}=1 / 3$ is

$$
\mathcal{U}_{A p, q}\left(p_{p}^{*}=1 / 3, q\right)=\frac{2}{3},
$$

and is independent of $q$. By symmetry, the same applies for players B utility for $q_{p}^{*}=2 / 3$

$$
\mathcal{U}_{B p, q}\left(p, q_{p}^{*}=2 / 3\right)=\frac{2}{3} .
$$

The expected utilities of the players when the opponent plays his mixed Nash strategy $p_{N}^{*}=q_{N}^{*}=1 / 3$ are

$$
\mathcal{U}_{A p, q}\left(p, q_{N}^{*}=1 / 3\right)=\mathcal{U}_{B p, q}\left(p_{N}^{*}=2 / 3, q\right)=\frac{2}{3},
$$

and these values do not depend on the own action, and the expected utilities are maximized when the opponent plays mixed Nash. Also note that the expected utilities for the mixed periodic strategy take also their maximum values. The utilities corresponding to the periodic strategies and to the mixed Nash equilibria coincide.

The disadvantage of the mixed Nash strategy, compared to the mixed periodic strategy, is that in order for the expected utility to be maximized, the opponent has to play Nash. This renders all the strategies of the player optimal. However, this does not happen in the periodic mixed strategy case, where when a player plays his own periodic mixed strategy, the expected utility is maximized, regardless of what the other player plays. We observe furthermore that if player A plays his own Nash mixed strategy $p_{N}^{*}=2 / 3$, his expected utility is

$$
\mathcal{U}_{A p, q}\left(p_{N}^{*}=2 / 3, q\right)=\frac{1}{3}+q .
$$

Accordingly, the expected utility of player B for $q_{N}^{*}=1 / 3$ is

$$
\mathcal{U}_{B p, q}\left(p, q_{N}^{*}=1 / 3\right)=\frac{4}{3}-p .
$$

These utilities depend on what the other player plays, and thus the mixed strategies of each player do not render the corresponding payoff robust against the opponent's strategies.

In contrast, the mixed periodic strategies render the corresponding payoffs robust to what the opponent chooses, and in addition these maximize the expected utility functions. This result is very intriguing, since the mixed periodic strategies we found, namely $\left(p_{p}^{*}=1 / 3, q_{p}^{*}=2 / 3\right)$ are not rationalizable actions. Nevertheless, we have seen that the player who adopts these strategies always achieves equal or larger 
payoff in comparison to the mixed Nash payoff, regardless of what his opponent eventually plays (we assume here $0<p, q<1$ ).

\subsubsection{Second type of games}

We now turn to games that satisfy

$$
\begin{aligned}
& p_{p}^{*}=1-q_{N}^{*}, \\
& q_{p}^{*}=1-p_{N}^{*} .
\end{aligned}
$$

These conditions render the corresponding expected utilities robust against the opponent strategies. In addition, condition (56) implies

$$
\mathcal{U}_{A p, q}\left(a_{1}, b_{1}\right)=\mathcal{U}_{A p, q}\left(a_{2}, b_{2}\right), \quad \mathcal{U}_{B p, q}\left(a_{1}, b_{1}\right)=\mathcal{U}_{B p, q}\left(a_{2}, b_{2}\right) .
$$

Let us illustrate this result for Game 2 in Table 9. We have two pure Nash equilibria, $\left(a_{1}, b_{2}\right)$ and $\left(a_{2}, b_{1}\right)$, which at the same time are periodic, and the mixed Nash equilibrium $\left(p_{N}^{*}=\frac{5}{6}, q_{N}^{*}=\frac{48}{49}\right)$. The mixed periodic strategy of $\mathrm{A}$ is $p_{p}^{*}=1 / 49$ according to $(56)$, and that for $\mathrm{B}$ is $q_{p}^{*}=1 / 6$. The expected utility for player $\mathrm{A}$ at $p_{p}^{*}=1 / 49$ is

$$
\mathcal{U}_{A p, q}\left(p_{p}^{*}=1 / 49, q\right)=\frac{146}{49},
$$

which is again independent of $q$. Similarly, that of B for $q_{p}^{*}=1 / 6$ is

$$
\mathcal{U}_{B p, q}\left(p, q_{p}^{*}=1 / 6\right)=\frac{35}{6} .
$$

Again, these coincide with the expected utilities resulting from playing Nash,

$$
\mathcal{U}_{A p, q}\left(p, q_{N}^{*}=48 / 49\right)=\frac{146}{49}, \quad \mathcal{U}_{B p, q}\left(p_{N}^{*}=5 / 6, q\right)=\frac{35}{6} .
$$

Again, the mixed Nash payoffs are independent of the own action and the expected utilities are maximized when the opponent plays his mixed Nash strategy. And again, the expected utilities for the mixed periodic strategy take their maximum values, which are equal to the ones obtained for the Nash strategies. However, if player A plays for instance his own Nash mixed strategy $p_{N}^{*}=5 / 6$, his expected utility is

$$
\mathcal{U}_{A p, q}\left(p_{N}^{*}=5 / 6, q\right)=-\frac{239}{6} q+42
$$

Table 9. Mixed strategies game 2 .

\begin{tabular}{lll}
\hline & $b_{1}$ & $b_{2}$ \\
\hline$a_{1}$ & 2,5 & 50,6 \\
$a_{2}$ & 3,10 & 2,5 \\
\hline
\end{tabular}


while the expected utility for player B, when he plays $q_{N}^{*}=48 / 49$, is

$$
\mathcal{U}_{B p, q}\left(p, q_{N}^{*}=1 / 3\right)=\frac{485-239 p}{49} .
$$

Again, these utilities depend on what the opponent plays, in contrast to the mixed periodic strategies. And again, the two types of utilities coincide.

As a general remark for the types of games appearing in Tables 8 and 9, we should note that $U_{i}\left(p_{p}, q_{p}\right)=U_{i}\left(p_{N}, q_{N}\right)$, whenever both the Nash and the periodicity solution concepts require a mixed strategy. This can also be seen by looking Eqs. (58)-(60).

Since in game theory, one is often more interested in pure than in mixed solutions, we now look at another game in Table 10, For this game, by applying the periodicity algorithm, we find that the mixed periodic payoffs are

$$
\mathcal{U}_{A p, q}\left(p_{p}^{*}=1 / 2, q_{p}^{*}=1 / 2\right)=\frac{1}{2}, \quad \mathcal{U}_{B p, q}\left(p_{p}^{*}=1 / 2, q_{p}^{*}=1 / 2\right)=\frac{3}{2},
$$

and the corresponding mixed Nash payoffs are

$$
\mathcal{U}_{A p, q}\left(p_{N}^{*}=1 / 2, q_{N}^{*}=1 / 2\right)=\frac{1}{2}, \quad \mathcal{U}_{B p, q}\left(p_{N}^{*}=1 / 2, q_{N}^{*}=1 / 2\right)=\frac{3}{2},
$$

so the two solution concepts yield the same mixed payoffs. However, the pure Nash equilibrium is $\left(a_{2}, b_{2}\right)$, so the player B gets a higher payoff for the pure Nash strategy. The same would apply for player A if we transposed the payoffs. Therefore, sometimes periodicity is better, while at other times Nash is better. This seems to be a generic characterization which covers more types of games, compared with the first and second type games discussed earlier in this section.

We now turn to games where the expected periodic utilities are actually higher than those from mixed Nash.

\subsubsection{When periodicity is better than Nash mixed strategies: The case of collective action games and prisoner dilemma type of games}

In this section, we shall treat a class of games that includes the "Pure Public Good" games. Let us recall an example. Suppose that two oil companies want to extract oil near some island and transfer it to international markets. For that, a pipeline is needed the government's public policy allows only one pipeline, so both companies must share the pipeline, when it is constructed. Both companies will benefit from it, but the question is who is going to fund the construction of this pipeline.

Table 10. Alternative example.

\begin{tabular}{ccc}
\hline & $b_{1}$ & $b_{2}$ \\
\hline$a_{1}$ & 4,0 & $-1,-1$ \\
$a_{2}$ & $-3,1$ & 0,4 \\
\hline
\end{tabular}


Table 11. Mixed strategies game 3.

\begin{tabular}{ccc}
\hline & $b_{1}$ & $b_{2}$ \\
\hline$a_{1}$ & 4,4 & $-1,6$ \\
$a_{2}$ & $6,-1$ & 0,0 \\
\hline
\end{tabular}

Such games are inherent to problems of collective action [26]. In this kind of games, the actions that yield better payoffs for the players do not belong to the set of best private interest actions of the players, or more formally, the Pareto optimal outcome is not necessarily the Nash equilibrium.

The pipeline project, like any Pure Public Good game, has two characteristic properties that the benefits are nonexcludable and nonrival. Such a game can be represented in matrix form in Game 3 in Table 11 (taken from [26]). The Nash equilibrium is $\left(a_{2}, b_{2}\right)$. The payoffs depend on the quality and the time that it takes to materialize the project. Obviously, the optimal action for both players is not to participate, no matter what the other player does, that is, to act as a "free rider". In contrast, the Pareto optimum is achieved when the strategy $\left(a_{1}, b_{1}\right)$ is adopted by both players.

The social optimal is always achieved when the total sum of the players payoffs is maximized, but this requires a cooperative way of thinking. Using mixed periodic strategies, we now want to analyze the game within a noncooperative perspective. Let us analyze the mixed strategies that this game has. There is no mixed Nash equilibrium, but only the pure Nash strategy $\left(a_{2}, b_{2}\right)$. The expected utilities of the two players for $p=p_{N}^{*}=0$, and $q=q_{N}^{*}=0$ are both

$$
\mathcal{U}_{A p, q}\left(p_{N}^{*}=0, q_{N}^{*}=0\right)=\mathcal{U}_{B p, q}\left(p_{N}^{*}=0, q_{N}^{*}=0\right)=0 .
$$

For identifying mixed periodic strategies, we maximize the expected utility of player A with respect to $q$ and that of player B with respect to $p$. This results in the pure strategies $p_{p}^{*}=1$ and $q_{p}^{*}=1$. The expected utilities of both players are maximized for this periodic strategy,

$$
\mathcal{U}_{A p, q}\left(p_{p}^{*}=1, q_{p}^{*}=1\right)=\mathcal{U}_{B p, q}\left(p_{p}^{*}=1, q_{p}^{*}=1\right)=4 .
$$

Hence, in this case, the social optimum strategy is realized by periodic strategies, although we have used a noncooperative method in terms of a self-maximization procedure for each player. The fact that the two outcomes, that is, noncooperative and cooperative ones, coincide depends on the particular details of this game. But the important insight is that the noncooperative optimization procedure underlying the periodic strategies yields a higher payoff than the Nash strategies.

We will further analyze this type of games, by exploiting another example, Game 4 in Table 12, which again is a collective action game. The mixed Nash equilibrium is $p_{N}^{*}=\frac{3}{4}, q_{N}^{*}=\frac{3}{4}$. If we maximize A's expected utility subject to $q$ we get $\frac{\partial \mathcal{U}_{A}}{\partial q}=-2<$ $0 \forall q$. Hence, the expected utility is maximized when $q=q_{p}^{*}=0$, since the utility is 
Table 12. Mixed strategies game 4 .

\begin{tabular}{lll}
\hline & $b_{1}$ & $b_{2}$ \\
\hline$a_{1}$ & 0,0 & 6,1 \\
$a_{2}$ & 1,6 & 3,3 \\
\hline
\end{tabular}

monotonically decreasing with respect to $q$. Correspondingly, maximizing B's expected utility with respect to $p$ we get $\frac{\partial \mathcal{U}_{B}}{\partial p}=-2<0 \forall p$, and B's expected utility is maximized when $p_{p}^{*}=0$. Therefore, the periodic strategies are $p_{p}^{*}=0, q_{p}^{*}=0$. For the periodic strategies, $\mathcal{U}_{B p, q}\left(p_{p}^{*}=0, q_{p}^{*}=0\right)=3$ and $\mathcal{U}_{A p, q}\left(p_{p}^{*}=0, q_{p}^{*}=0\right)=3$ while for the Nash strategies we get $\mathcal{U}_{A p, q}\left(q_{N}^{*}=3 / 4, p_{N}^{*}=3 / 4\right)=2.25$ and $\mathcal{U}_{B p, q}\left(q_{N}^{*}=3 / 4, p_{N}^{*}=3 / 4\right)=2.25$.

Hence, the periodic strategy, which is pure in this game, again yields higher payoffs for both players than the Nash strategy.

In conclusion, this provides a possible solution to the question why the players should play socially optimal strategies, instead of individually optimal ones, through a strictly noncooperative scheme.

\section{Two-Player Simultaneous Strategic Form Games with a Continuum Set of Strategies}

In this section, we shall study the implications of the periodic strategies algorithm for the case of two player games with continuous strategies for the players. We shall consider strategic form, simultaneous, symmetric games with quadratic payoffs. Many examples from the economics literature belong to this class of games, such as the Cournot and Bertrand duopoly, provision of public good and search games [27]. while the periodic strategies algorithm does not yield as interesting results as in the collective action games, we shall nevertheless present it in order to explore possible applications of the algorithm.

We consider a game with two players $I=1,2$, for which a continuum set of strategies is available for each player and where the payoffs are of the following form:

$$
\begin{aligned}
& u_{1}(x, y)=a_{1} x+a_{2} y+a_{3} x y+a_{4} x^{2}+a_{5} y^{2} \\
& u_{2}(x, y)=b_{1} x+b_{2} y+b_{3} x y+b_{4} x^{2}+b_{5} y^{2}
\end{aligned}
$$

where $x, y \in \mathbb{R}^{+}$. In both cases, the parameters $a_{5}, b_{5}$ are assumed to be negative, to make the payoffs concave in the own strategy. For simplicity, we assume that the game is symmetric, that is $a_{i}=b_{i}$ for all $i$. For the Nash equilibria, the following equations must be solved simultaneously:

$$
\frac{\partial u_{1}}{\partial x}=0, \quad \frac{\partial u_{2}}{\partial y}=0 .
$$


For the periodic strategies, we have to solve simultaneously:

$$
\frac{\partial u_{1}}{\partial y}=0, \quad \frac{\partial u_{2}}{\partial x}=0
$$

In order for the critical points $\left(x_{N}, y_{N}\right)$ of equations (68) to be maxima, the following two conditions have to be satisfied:

$$
\begin{array}{ll}
D_{1}=\frac{\partial^{2} u_{1}}{\partial x^{2}} \frac{\partial^{2} u_{1}}{\partial y^{2}}-\frac{\partial^{2} u_{1}}{\partial y \partial x}>0, & \left.\frac{\partial^{2} u_{1}}{\partial x^{2}}\right|_{x_{N}, y_{N}}<0, \\
D_{2}=\frac{\partial^{2} u_{2}}{\partial x^{2}} \frac{\partial^{2} u_{2}}{\partial y^{2}}-\frac{\partial^{2} u_{2}}{\partial y \partial x}>0, & \left.\frac{\partial^{2} u_{2}}{\partial x^{2}}\right|_{x_{N}, y_{N}}<0 .
\end{array}
$$

In the case of general quadratic games, the above conditions become

$$
\begin{array}{ll}
D_{1}=-a_{3}^{2}+4 a_{4} a_{5}>0, & a_{4}<0, \\
D_{2}=-b_{3}^{2}+4 b_{4} b_{5}>0, & b_{4}<0 .
\end{array}
$$

The conditions for maxima of the periodic strategies algorithm are

$$
\begin{array}{ll}
D_{1}=-a_{3}^{2}+4 a_{4} a_{5}>0, & a_{5}<0 \\
D_{2}=-b_{3}^{2}+4 b_{4} b_{5}>0, & b_{5}<0 .
\end{array}
$$

For symmetric games, the conditions simplify:

$$
D_{1}=-a_{3}^{2}+4 a_{4} a_{5}>0, \quad a_{4}<0, \quad a_{5}<0 .
$$

These last conditions are satisfied for all quadratic games that satisfy the convexity condition imposed above. The Nash equilibria and the "periodic" points are:

$$
\begin{array}{lll}
x=-\frac{-2 a_{1} b_{5}-a_{3} b_{2}}{-a_{3} b_{3}+4 a_{4} b_{5}}, & y=-\frac{-2 a_{4} b_{2}-a_{1} b_{3}}{-a_{3} b_{3}+4 a_{4} b_{5}}, & \text { Nash, } \\
x=-\frac{-2 a_{5} b_{1}+a_{2} b_{3}}{a_{3} b_{3}-4 a_{5} b_{4}}, \quad y=-\frac{a_{3} b_{1}-2 a_{2} b_{4}}{a_{3} b_{3}-4 a_{5} b_{4}}, \quad \text { Periodic. }
\end{array}
$$

We shall now consider two examples of continuous, symmetric quadratic games, the "Cournot Duopoly" game and the "Provision of Public Good" game.

\subsection{Cournot duopoly}

The Cournot duopoly quadratic game has the following form:

$$
\begin{aligned}
& u_{1}(x, y)=(P-A(x+y)) x-\left(B x-M x^{2}\right), \\
& u_{2}(x, y)=(P-A(x+y)) y-\left(B y-M y^{2}\right) .
\end{aligned}
$$

Thus, in (67) (with $a_{i}=b_{i}$ ), we put

$$
a_{1}=P-B, \quad a_{3}=-A, \quad a_{4}=-A+M, \quad a_{2}=a_{5}=0 .
$$


The Nash equilibrium is obtained by putting $\frac{\partial u_{1}}{\partial x}=0=\frac{\partial u_{2}}{\partial y}$, resulting in

$$
x_{N}^{*}=y_{N}^{*}=\frac{P-B}{3 A-2 M},
$$

and the corresponding utilities are:

$$
u_{1}\left(x_{N}^{*}, y_{N}^{*}\right)=u_{2}\left(x_{N}^{*}, y_{N}^{*}\right)=\frac{(P-B)^{2}(A-M)}{(3 A-2 M)^{2}}
$$

In contrast, for the periodic equilibrium, we need $\frac{\partial u_{2}}{\partial x}=0=\frac{\partial u_{1}}{\partial y}$, resulting in

$$
x_{p}^{*}=y_{p}^{*}=0 \text { and } u_{1}\left(x_{p}^{*}, y_{p}^{*}\right)=u_{2}\left(x_{p}^{*}, y_{p}^{*}\right)=0 .
$$

Thus, if $A>M$, the Nash equilibrium results in higher utilities for the players. This game, however, is degenerate in the sense that when computing the periodic optimum for A by putting $0=\frac{\partial u_{2}}{\partial x}=-A x$, this does not determine the optimal value of the opponent's strategy $y$. In fact, for this game, in (67), the coefficient $a_{5}$ of the quadratic term vanishes by (76), and this causes the degeneracy.

\subsection{Provision of public good games}

The Provision of Public Good quadratic game has the following form:

$$
\begin{aligned}
& u_{1}(x, y)=A(x+y)-C x-B(x+y)^{2}, \\
& u_{2}(x, y)=A(x+y)-C y-B(x+y)^{2} .
\end{aligned}
$$

The Nash equilibria have to satisfy

$$
x_{N}^{*}+y_{N}^{*}=\frac{A-C}{2 B}
$$

with utilities

$$
u_{1}\left(x_{N}^{*}, y\right)=\frac{(A-C)^{2}}{4 B}+C y, \quad u_{2}\left(x, y_{N}^{*}\right)=\frac{(A-C)^{2}}{4 B}+C x .
$$

For the periodic equilibria, we get

$$
x_{p}^{*}+y_{p}^{*}=\frac{A}{2 B}
$$

with utilities

$$
u_{1}\left(x_{p}^{*}, y\right)=\frac{A^{2}-2 A C}{4 B}+C y, \quad u_{2}\left(x, y_{p}^{*}\right)=\frac{A^{2}-2 A C}{4 B}+C x .
$$

Thus, the periodic utilities are smaller than the Nash ones, since

$$
u_{1}\left(x_{p}^{*}, y\right)=u_{1}\left(x_{N}^{*}, y\right)-\frac{C^{2}}{4 B}, \quad u_{2}\left(x, y_{p}^{*}\right)=u_{2}\left(x, y_{N}^{*}\right)-\frac{C^{2}}{4 B},
$$

unless $C=0$. At the periodic equilibrium, the players invest too much, as they ignore their own costs $C$. Again, however, this is a special case, because of the symmetry of the game. 


\section{Concluding Remarks}

We have presented an intrinsic property of multiplayer, finite, simultaneous, strategic form games, which we called periodicity of strategies. We studied the periodicity concept in finite action games and proved that every finite action two player strategic form game has at least one periodic action. Moreover, we proved that the set of periodic strategies is set stable under the map $\mathcal{Q}$. An action of some player $x_{i}$ is periodic if the operator $\mathcal{Q}$ satisfies $\mathcal{Q}^{n_{i}} x_{i}=x_{i}$, where $n_{i}$ is the periodicity number. When mixed strategies are taken into account, the period length reduces to 1 . While those equilibria are different from the Nash ones, the resulting payoffs for the players are the same, or in some cases, even higher than the Nash payoffs. Importantly, the periodic mixed strategy gives outcomes for each player which do not depend on what the opponent will play, in contrast to the Nash mixed strategy, where when the opponent plays equilibrium, the own action does not matter. Thus, we have found a solution concept that in that regard is preferable to the Nash equilibrium. More generally, we have compared our periodic strategies to the rationalizable strategies of Bernheim [7] and Pearce [8]. Moreover, the application of the algorithm to collective action games gives another interesting result. We were able to demonstrate that the social optimum strategy can be played by adopting a noncooperative thinking.

Also, the periodic equilibria can be identified by a straightforward algorithm. The next step would be the inclusion of mixed strategies in multiplayer games. In that case, the situation will become more complex because the players could form coalitions. Periodicity then has to be reconsidered under this perspective. Also, one may wish to study Bayesian games with imperfect information from the perspective of our periodicity paradigm.

\section{References}

[1] Fudenberg, D. and Tirole, J., Game Theory (MIT Press, US, 1991).

[2] Wright, J. R. and Leyton-Brown, K., Beyond Equilibrium: Predicting Human Behavior in Normal-Form Games. Proceeding of the Twenty-Fourth AAAI Conference on Artificial Intelligence (AAAI-10) (2010), pp. 901-907.

[3] M. Mariotti, A model of agreements in strategic form games, J. Econ. Theory 74(1) (1997) 196-217.

[4] Osborne, M. J., An Introduction to Game Theory (Oxford University Press, UK, 2004).

[5] Geanakoplos, J., Common knowledge, J. Econ. Perspect. 6 (1992) 53-82.

[6] John von, N. and Oskar, M., Theory of Games and Economic Behavior (Princeton University Press, US, 2004).

[7] Bernheim, B. D., Rationalizable strategic behavior, Econometrica 52 (1984) 1007.

[8] Pearce, D., Rationalizable strategic behavior and the problem of perfection, Econometrica 52 (1984) 1029-1050.

[9] Myerson, R. B., Refinements of the Nash equilibrium concept, Int. J. Game Theory 7 (1978) 73-80.

[10] Battigalli, P., On rationalizability in extensive games, J. Econ. Theory 74 (1997) 40-61. 
[11] Pierpaolo B., Rationalizability in infinite, dynamic games with incomplete information, Res. Econ. 57 (1996) 1-38.

[12] Battigalli, P., Strategic independence and perfect Bayesian equilibria, J. Econ. Theory 70 (1996) 201-234.

[13] Battigalli, P. and Siniscalchi M., Hierarchies of conditional beliefs, and interactive epistemology in dynamic games, J. Econ. Theory 88 (1999) 188-230.

[14] Battigalli, P. and Siniscalchi M., Strong belief and forward induction reasoning, J. Econ. Theory 106 (2002) 356-339.

[15] Blume, L. E., Brandenburger, A. and Dekel E., Lexicographic probabilities and choice under uncertainty, Econometrica 59 (1991a) 61-79.

[16] Blume, L. E., Brandenburger, A. and Dekel E., Lexicographic probabilities and equilibrium refinements, Econometrica 59 (1991b) 81-98.

[17] Dekel, E. and Fudenberg D., Rational behavior and payoff uncertainty, J. Econ. Theory 52 (1990) 243-267.

[18] Rubinstein, A., Comments on the interpretation of game theory, Econometrica 59 (1991) 909-924.

[19] Schuhmacher, F., Proper rationalizability and backward induction, Int. J. Game Theory 28 (1999) 599-615.

[20] Asheim, G. B., Proper rationalizability in lexicographic beliefs, Int. J. Game Theory 30 (2001) 453-478.

[21] Epstein, L. and Wang T., "Beliefs about beliefs" without probabilities, Econometrica 64 (1996) 1343.

[22] Reny, P. J., Rationality in extensive-form games, J. Econ. Perspect. 6 (1992) 103.

[23] Stalnaker, R., Belief revision in games: Forward and backward induction, Math. Soci. Sci. 36 (1998) 31-56.

[24] van Damme, E. (1984). A relation between perfect equilibria in extensive form games and proper equilibria in normal form games, Int J. Game Theory 13, 1-13.

[25] Srihari G. and Robert W., On forward induction, Econo. Econ. Soci. 77(1) (2009) 1-28.

[26] Avinash K. D. and Susan S., Games of Strategy, 2nd edn. (W. W. Norton, US, 2004).

[27] Moulin, H., Ray, I. and Gupta, S. S., Improving Nash by Correlation in Quadratic Potential Games (2012). 